\title{
Polarisation pulling in Brillouin optical time-domain analysers
}

\author{
Marcelo A. Soto*a, Moshe Tur ${ }^{\mathrm{a}, \mathrm{b}}$, Alexia Lopez-Gil ${ }^{\mathrm{c}}$, Miguel Gonzalez-Herraez ${ }^{\mathrm{c}}$, Luc Thévenaz ${ }^{\mathrm{a}}$ \\ ${ }^{a}$ EPFL Swiss Federal Institute of Technology, Inst. ELE, SCI STI LT, 1015 Lausanne, Switzerland \\ ${ }^{b}$ School of Electrical Engineering, Tel Aviv University, Ramat Aviv 69978, Israel \\ 'Dep. de Electrónica, Universidad de Alcalá, Edificio Politécnico, 28805 Alcala de Henares, Spain \\ *E-mail: marcelo.soto@epfl.ch
}

\begin{abstract}
The impact of Brillouin scattering on the polarisation of pump and probe in a Brillouin optical-time domain analyser is investigated. Experimental results indicate that the Brillouin interaction integrated along the entire sensing fibre produces a polarisation pulling force that changes the pump polarisation. Although this force can be compensated to first order by dual-sideband probes, the Brillouin gain/loss experienced by probe bands induce a net pulling that leads to uncompensated polarisation fading and reduced signal-to-noise ratio. While the use of two simultaneous orthogonally-polarised probes alleviates fading issues, spectral distortions originate from the coupling between Brillouin gain and polarisation pulling.
\end{abstract}

Keywords: Optical fibre sensor, stimulated Brillouin scattering, distributed fibre sensor, polarisation

\section{INTRODUCTION}

For several years distributed fibre sensing based on Brillouin optical-time domain analysis (BOTDA) has attracted the attention of different sectors, thanks to their unique ability to perform accurate distributed strain and temperature sensing over several tens of kilometres ${ }^{1,2}$. The measurement signal-to-noise ratio (SNR) has been identified as the most relevant parameter ultimately determining the sensor performance ${ }^{2}$, and although the limitations imposed by nonlinear effects and noise can be partially overcome with sophisticated techniques ${ }^{1}$, there still exists another unexplored limitation imposed by polarisation issues. This is generally screened by the noise present in conventional BOTDA measurements. In particular, the impact of stimulated Brillouin scattering (SBS) on the state of polarisation (SOP) of the interacting pump and probe has not yet been addressed by the fibre sensing community. Only Ref. 3 has provided an analysis of the impact that SBS has on the SOP of interacting continuous waves (CW); indicating that the SOPs of CW pump and signal can mutually influence each other as a result of a strong polarisation pulling force ${ }^{3}$. Although in the case of BOTDA sensing the pump is pulsed and the local Brillouin interaction is very small, the impact of SBS from the probe onto the pump SOP turns out to be integrated along several tens of kilometres, making polarisation pulling a potentially significant issue. Interestingly, the impact of polarisation pulling on the performance of BOTDA sensors has never been addressed in the state-of-the-art.

This paper presents, for the first time to the best of our knowledge, a study on the impact of SBS on the polarisation of the pump as it interacts with the probe along a BOTDA sensor. A theoretical analysis indicates that, even though the SBS interaction is locally weak and has a minor effect on the probe SOP, the pump SOP is indeed influenced by the probe SOP. This polarisation pulling effect is experimentally analysed for different spatial resolutions and pump/probe powers. Results show that the use of a polarisation switch to compensate for polarisation fading generates different polarisation pulling forces, which lead to traces with strong residual uncompensated fading, thereby potentially compromising the SNR. The study is also extended to the case of using orthogonally-polarised probes, indicating that although polarisation fading can be significantly reduced, serious distortions in the measured gain spectrum are expected as a result of polarisation pulling.

\section{POLARISATION PULLING IN BOTDA SENSORS}

In a BOTDA sensor, a pulsed pump interacts with a counter-propagating CW probe by means of an SBS-induced acoustic wave $^{1,2}$. When the pump-probe frequency offset matches the local Brillouin frequency of the fibre, power is locally transferred between pump and probe $e^{1-4}$. This exchange of photons does not only lead to amplification or attenuation of the pump and probe, but also affects their state of polarisation. As a result, the evolution of the pump and probe SOPs along the sensing fibre turns out to depend not only on the fibre birefringence, as generally assumed, but also on the local SBS interaction taking place at each fibre location. In the case of a $\mathrm{CW}$ pump, propagating against a $\mathrm{CW}$ lower-frequency probe (- attenuating the pump) or CW higher-frequency probe (+ amplifying the pump), the pump SOP evolution is given by

\footnotetext{
25th International Conference on Optical Fiber Sensors, edited by Youngjoo Chung, Wei Jin,

Byoungho Lee, John Canning, Kentaro Nakamura, Libo Yuan, Proc. of SPIE Vol. 10323,

103239L · () 2017 SPIE · CCC code: 0277-786X/17/\$18 · doi: 10.1117/12.2267649
} 


$$
\frac{d}{d z} \hat{s}_{\text {pump }}(z)=\vec{\beta}(z) \times \hat{s}_{\text {pump }}(z) \pm \frac{\gamma_{0}}{2} P_{\text {probe }}(z)\left[\hat{s}_{\text {probe }}^{ \pm}(z)-\left(\hat{s}_{\text {probe }}^{ \pm}(z) \cdot \hat{s}_{\text {pump }}(z)\right) \hat{s}_{\text {pump }}(z)\right],
$$

where $\hat{s}_{\text {pump }}(z)$ and $\hat{s}_{\text {probe }}^{ \pm}(z)$ are the normalised Stokes vectors describing the polarisations of the pump and attenuating/ amplifying probes, $\vec{\beta}(z)$ is a three-dimensional vector describing the fibre birefringence in the Stokes space, $\gamma_{0}(>0)$ is the Brillouin gain per unit length per unit power and $P_{\text {probe }}(z)$ is the local power of the probe (note that unlike in Ref. 3, here the pump propagates in the $+z$ direction). The last term of Eq. (1) represents a pulling of the pump SOP towards the SOP of the amplifying probe $\left(\hat{s}_{\text {probe }}^{+}\right)$, while for the attenuating probe it represents a repulsion, i.e. pulling towards an SOP orthogonal to that of the attenuating probe $\left(-\hat{s}_{\text {probe }}^{-}\right)^{3}$. Thus, considering the sign of the Brillouin interaction in Eq. (1), the use of a two-sideband probe is expected to compensate not only the gain/attenuation on the pump ${ }^{4}$, but also the polarisation pulling forces. This is because the pump amplification induced by the high-frequency probe sideband attracts the pump polarisation towards the probe polarisation, while the pump attenuation induced by the low-frequency probe repels the pump polarisation away from the probe polarisation. Unfortunately, it will be shown below that a net pulling effect is observed in the real implementation (since sidebands never show perfectly equal powers). Although small, this net pulling can have a serious detrimental impact on the performance of some BOTDA implementations, especially when using a polarisation switch for polarisation fading compensation. This is because using a polarisation switch requires sequentially measuring time-domain traces with two orthogonal probe SOPs (or, equivalently, two orthogonal pumps). However since the two traces are sequentially acquired, the residual polarisation pulling forces acting on the pump pulse have different directions in each case. Thus, for instance, even if two perfectly orthogonally-polarised probes are used in the measurements, the SBS interaction occurs with different pump SOPs in each case (as a result of pulling), leading to observable different gains (polarisation fading) that do not fully compensate when the two time-domain traces are summed. It is important to notice that the probe signal reaching the photodetector at a given time $t$ interacts with the pump pulse only along a short fibre section (only along the pulse length), and therefore a very minor effect on the probe polarisation is expected under typical operating conditions. This is also a consequence of the weak local SBS interaction (typically $1 \%$ gain/loss for $1 \mathrm{~m}$ spatial resolution), which is required for the correct operation of a long-range BOTDA sensor. On the contrary, the pump pulse interacts with the $\mathrm{CW}$ probe along the entire sensing fibre, and therefore a significant impact on the pump SOP is observed as a result of the net SBS interaction (integrated along the entire fibre).

\section{EXPERIMENTAL OBSERVATIONS}

To evaluate the impact of the above described polarisation pulling, a standard $50 \mathrm{~km}$-long BOTDA scheme has been implemented inserting a polarisation switch in the optical branch in which a probe with two equal-power sidebands is generated. At the far end of the sensing fibre (input for the probe signal), an optical circulator has been inserted to analyse the SOP of the output pump pulse, which has been measured using a polarisation analyser under 3 different scenarios: the pump SOP is first measured in the absence of probe, i.e. with no SBS interaction, and then after interacting with the two probe sidebands being launched into the fibre, first, at a given initial SOP (defined by the first polarisation state of the switch), and then, with the orthogonal SOP (second polarisation state of the switch).

Figure 1 shows the measured output pump SOP on the Poincare sphere without (blue crossed markers) and with (red markers) probe propagating along the fibre, using a pump power of $100 \mathrm{~mW}$ and a pulse width of $50 \mathrm{~ns}$, where the pumpprobe frequency offset has been set to maximise the Brillouin interaction. In particular, Fig. 1(a) shows the opposite polarisation pulling of the pump SOP as the common polarisation of the dual-sideband probes is switched between two orthogonal states of polarisation. While the effect of a weak probe of $-4 \mathrm{dBm} / \mathrm{sideband}$ on the pump SOP is minimal, significant and opposite SBS-induced pulling (quantified by the relative angle, $2 \varepsilon$, between the two Stokes vectors) is observed when increasing probe power, as indicated in Fig. 1(b) and 1(c) for probe powers of $0 \mathrm{dBm}$ and $+4 \mathrm{dBm}$ per sideband, respectively. Although results are presented here only for pump pulses of $50 \mathrm{~ns}$ and $100 \mathrm{~mW}$, similar relative angles have been experimentally obtained for different pump peak powers (ranging from $25 \mathrm{~mW}$ to $100 \mathrm{~mW}$ ) and different spatial resolutions (tested between 1 to 5 metres), indicating that the pulling of the pump SOP appears to be independent of the power and width of the pump pulse (at least in a small-gain operation, as typically required).

Results in Fig. 1 clearly demonstrate the impact that the pump-probe SBS interaction has on the pump polarisation. This polarisation pulling can lead to BOTDA traces having strong uncompensated polarisation fading, as shown in Fig. 2(a). An expanded view of the last $\sim 500 \mathrm{~m}$ of fibre is presented in Fig. 2(b) for probe powers ranging from $-4 \mathrm{dBm}$ to $+4 \mathrm{dBm}$ per sideband. As shown in the figure, the residual polarisation fading increase with distance as a result of the cumulative pulling experienced by the pump along its propagation, which is further augmented by the strong pulling effect induced by the higher probe power propagating close to the far fibre end. Note that the BOTDA trace in Fig. 2(a) has been measured using $2 \mathrm{k}$ averages per polarisation to virtually eliminate detection noise. Thus, the fading in the trace shown in Fig. 2(a) 

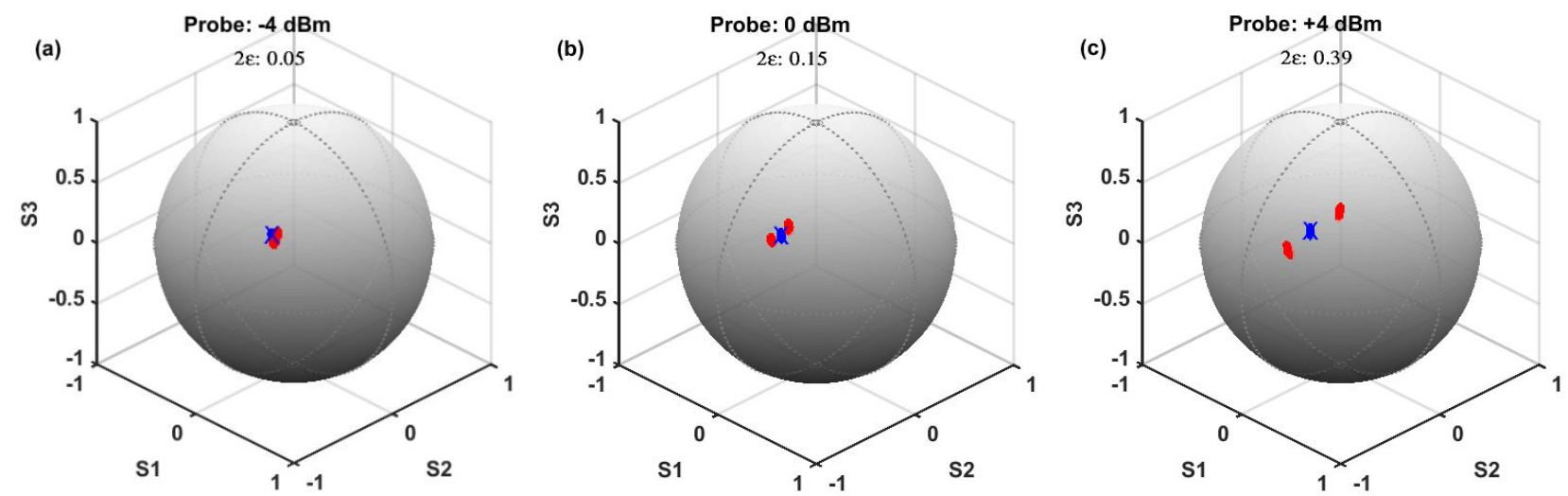

Figure 1. A Poincare sphere representation of the output pump SOPs measured without (blue markers) and with (red markers) SBS interaction. Each two red dot represents the pump SOP for one of two orthogonally but commonly polarised dual-sideband probes.

are deterministic in nature, as demonstrated in Fig. 2(b). This figure provides a reliable evaluation of the polarisation pulling effect, showing that, although the same mean gain value is obtained for all probe powers, the stronger the probe power, the larger the uncompensated polarisation fading. The main impact of this effect is the reduction of the trace amplitude at some fibre positions, decreasing the SNR of the measured local BGS, and therefore increasing the measurand uncertainty of the sensor. It is worth noticing that the residual fading shown in Fig. 2 occurs basically since the traces with the two orthogonal probe SOPs are separately measured, so that the pump SOP along the fibre turns out to be different for each case due to the different direction of the pulling forces, thus leading to gain traces that do not add up to the true gain.
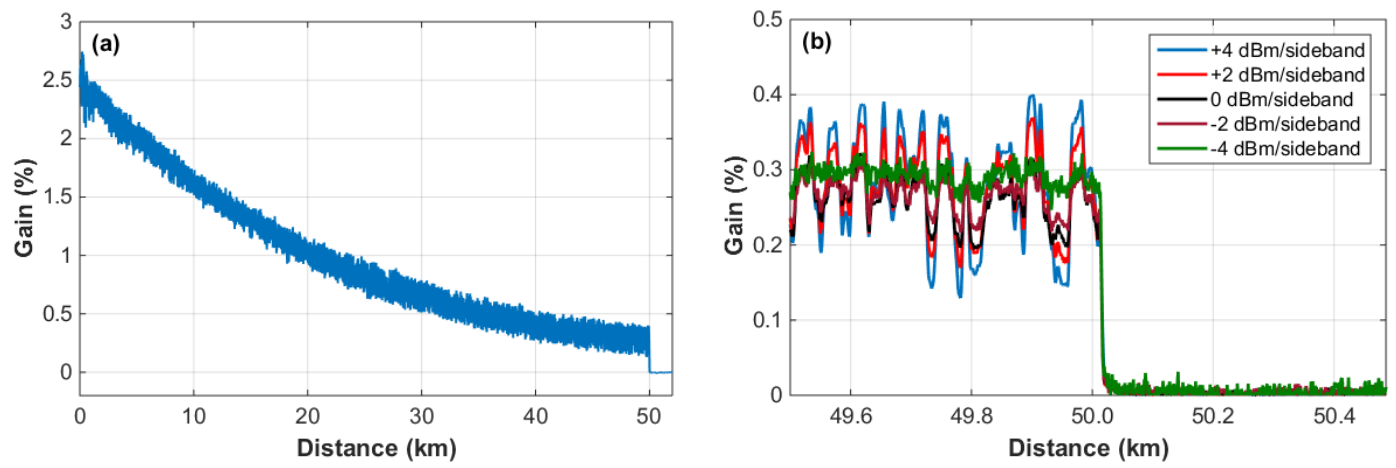

Figure 2. (a) $50 \mathrm{~km}$-long BOTDA trace showing strong residual uncompensated polarisation fading (probe power of $4 \mathrm{dBm} / \mathrm{sideband}$ )

(b) Traces along the last $\sim 500 \mathrm{~m}$ of fibre, showing the dependence of the residual fading amplitude on probe power.

To fully compensate polarisation fading, the two orthogonal probe SOPs must interact with the same pump SOP. This can be implemented by rotating the polarisation of one of the probe sidebands, so that the two orthogonally-polarised sidebands are simultaneously launched into the sensing fibre ${ }^{5}$. Time-domain traces can then be obtained using a balanced detector, in which each of the sidebands is launched into one of the two inputs of the detector ${ }^{5}$. Figure 3 shows the output pump SOPs (after $50 \mathrm{~km}$ ) measured without (blue markers) and with (red markers) simultaneous orthogonally-polarised probes. Compared to the use of parallel-polarised probe sidebands (see Fig. 1), results here prove that the use of orthogonal probes leads to a much stronger polarisation pulling force. This observation is fully consistent with Eq. (1): while for the parallel case $\hat{s}_{\text {probe }}^{+}=\hat{s}_{\text {probe }}^{-}$, for the orthogonal one $\hat{s}_{\text {probe }}^{+}=-\hat{s}_{\text {probe }}^{-}$, so that the two orthogonally-polarised probes contribute to pulling in the same direction. It is however important to notice that this strong pulling is not an issue since the two orthogonal probes interact simultaneously with the same local pump SOP, and therefore the trace measured at the output of the balanced detector is expected to be free of polarisation fading. This effect has been evaluated by analysing the longitudinal evolution of the residual polarisation fluctuations, quantified by the longitudinal standard deviation, which has been normalised by the local mean value of the trace to eliminate the fibre loss dependence of the SBS gain. Figure 4 shows the longitudinal standard deviation of the time-domain traces (i.e. calculated along the fibre using a moving window of 400 points) obtained with a probe power of $4 \mathrm{dBm} /$ sideband with the standard measurement method (i.e. using an orthogonal sequence of parallel-polarised probes) and using simultaneous orthogonal probe SOPs. It can be seen that the use of simultaneous orthogonally-polarised probes leads to a much better compensation of the polarisation fading along 

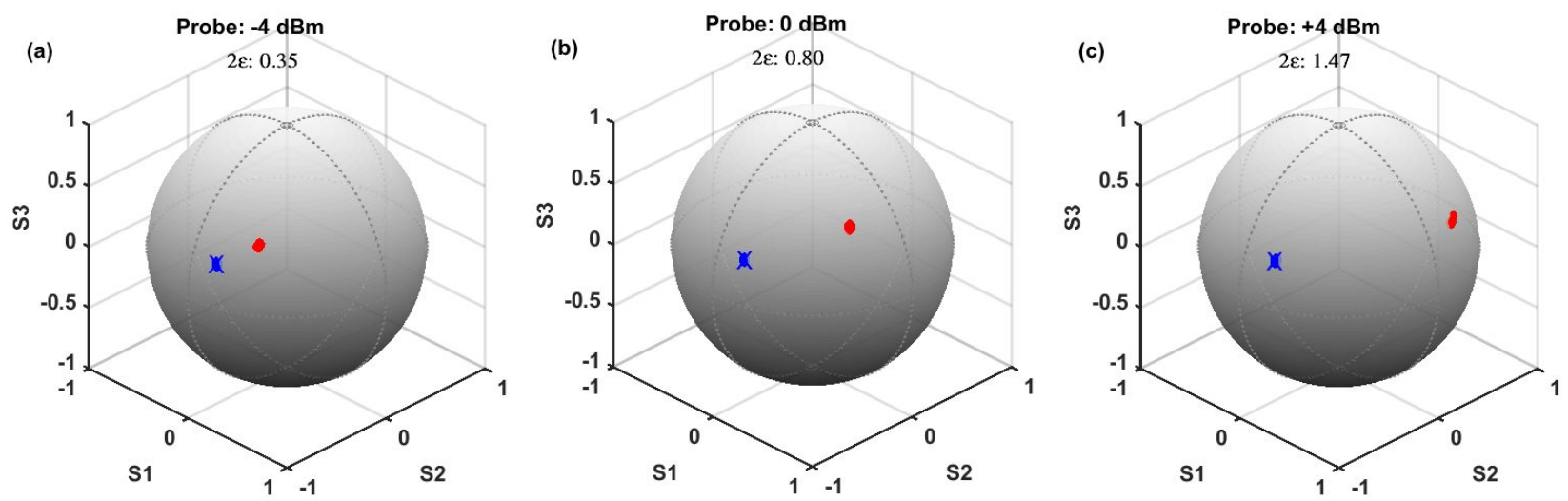

Figure 3. Pump SOPs measured without (blue markers) and with (red markers) SBS interaction using simultaneous orthogonal probes.

the entire sensing length. However, since the polarisation pulling is gain-dependent, this effect might have a strong detrimental impact on the BGS measurement. This is because the measured BGS now depends not only on the pump-probe frequency offset but is also affected by the dependence of polarisation pulling on gain strength. The net effect is a gradual alignment of the pump SOP with one of the sidebands, strongly impairing the depletion compensation inherent to the dual sideband scheme. This strong coupling between gain and polarisation pulling leads to BGS distortions that affect the frequency uncertainty of the measurements. A more complete evaluation of this spectral distortion and its detrimental impact on the performance of the sensor is still underway.

In conclusion, a polarisation pulling effect on the pump SOP has been experimentally observed in a BOTDA sensor, being significant enough to have a detrimental impact on the quality of the traces when

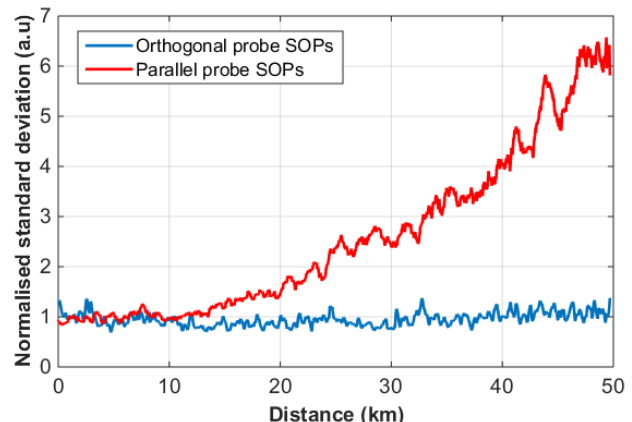

Figure 4. Longitudinal standard deviation (normalised by the local mean value) of traces measured with two simultaneous orthogonally-polarised probes (blue line) and with two independent orthogonal SOPs for two probe bands having the same polarisation (red line). using strong probe powers. This effect originates from probe powers that were originally slightly $(<1 \%)$ unbalanced on launch or made inevitably unequal by the very Brillouin interaction with the pump pulse. Some particular probing techniques may be more sensitive to this effect than others. Although the impact of uncompensated fading could be partially mitigated using orthogonally-polarised probes, this scheme results in strong spectral distortions, even at low probe powers, thus increasing substantially the measurand uncertainty. In addition, schemes using a (relatively-strong) single sideband probe would result in strong pump polarisation pulling since there is no second probe wave to compensate for the pulling force. Further research is underway to investigate techniques to mitigate polarisation pulling effects.

M. A. Soto and L. Thévenaz acknowledge the support from the Swiss Commission for Technology and Innovation (Project 18337.2 PFNM-NM). The work of M. Tur was supported in part by the Israel Science Foundation (grant No. 1380/12). The work of M. Gonzalez-Herraez was supported in part by the European Research Council through project U-FINE (Grant 307441).

\section{REFERENCES}

[1] Motil, A., Bergman, A, and Tur, M., "State of the art of Brillouin fiber-optic distributed sensing," Optics \& Laser Technology 78(A), 81-103 (2016).

[2] Soto, M. A., and Thévenaz, L., "Modeling and evaluating the performance of Brillouin distributed optical fiber sensors," Opt. Express 21(25), 31347-31366 (2013)

[3] Zadok, A., Zilka, E., Eyal, A., Thévenaz, L., and Tur, M., "Vector analysis of stimulated Brillouin scattering amplification in standard single-mode fibers," Opt. Express 16(26), 21692-21707 (2008)

[4] Thévenaz, L, Mafang, S. F., and Lin, J., "Effect of pulse depletion in a Brillouin optical time-domain analysis system," Opt. Express 21(12), 14017-14035 (2013)

[5] Lopez-Gil, A., et al., "Simple method for the elimination of polarization noise in BOTDA using balanced detection and orthogonal probe sidebands," J. of Lightwave Technol. 33(12), 2605-2610 (2015). 